\title{
Cortical mapping with navigated transcranial magnetic stimulation in low-grade glioma surgery
}

\author{
This article was published in the following Dove Press journal: \\ Neuropsychiatric Disease and Treatment \\ 2 May 2012 \\ Number of times this article has been viewed
}

\author{
Wellingson S Paiva' \\ Erich T Fonoff' \\ Marco A Marcolin² \\ Hector N Cabrera' \\ Manoel J Teixeira' \\ 'Division of Functional Neurosurgery, \\ Hospital das Clinicas, ${ }^{2}$ TMS \\ Laboratory of the Psychiatry Institute, \\ Hospital das Clinicas, University of \\ São Paulo School of Medicine, São \\ Paulo, Brazil
}

Correspondence: Wellingson S Paiva University of São Paulo, Eneas Aguiar Street, 255 Office 4080, São Paulo, Brazil 05403010

Tel +55 II 25486900

$\mathrm{Fax}+55$ || 25486906

Email wellingsonpaiva@yahoo.com.br

\begin{abstract}
Transcranial magnetic stimulation (TMS) is a promising method for both investigation and therapeutic treatment of psychiatric and neurologic disorders and, more recently, for brain mapping. This study describes the application of navigated TMS for motor cortex mapping in patients with a brain tumor located close to the precentral gyrus.

Materials and methods: In this prospective study, six patients with low-grade gliomas in or near the precentral gyrus underwent TMS, and their motor responses were correlated to locations in the cortex around the lesion, generating a functional map overlaid on three-dimensional magnetic resonance imaging (MRI) scans of the brain. To determine the accuracy of this new method, we compared TMS mapping with the gold standard mapping with direct cortical electrical stimulation in surgery. The same navigation system and TMS-generated map were used during the surgical resection procedure.

Results: The motor cortex could be clearly mapped using both methods. The locations corresponding to the hand and forearm, found during intraoperative mapping, showed a close spatial relationship to the homotopic areas identified by TMS mapping. The mean distance between TMS and direct cortical electrical stimulation (DES) was $4.16 \pm 1.02 \mathrm{~mm}$ (range: $2.56-5.27 \mathrm{~mm}$ ).

Conclusion: Preoperative mapping of the motor cortex with navigated TMS prior to brain tumor resection is a useful presurgical planning tool with good accuracy.
\end{abstract}

Keywords: transcranial magnetic stimulation, cortical mapping, brain tumor, motor cortex

\section{Introduction}

For centuries, scientists have strived to establish causal relationships between neural activity in a specific brain region and a particular behavioral or cognitive function. ${ }^{1-4}$ The first localization studies connecting anatomy and brain function were based on post-mortem analyses of brain lesions. ${ }^{1}$ Subsequently, functional brain mapping became possible during neurosurgical procedures by applying electrical stimulation directly to cortical tissue, evoking behavioral responses. ${ }^{1,4}$ With the advent of new functional brain imaging techniques in the past two decades, noninvasive mapping during event-related activation of select brain areas became possible, but unlike during invasive electrical stimulation, no direct cause-effect relationship could be established. ${ }^{5,6}$

Cortical locations corresponding to neurologic functions can vary significantly among individuals. In addition, some brain lesions distort the underlying anatomical features, hindering the localization of certain key intraoperative locations. However, determining the exact cortical area responsible for a specific function is essential for safe and effective resection. ${ }^{7}$ As the extent of tumor lesion resection is directly related to outcome, the surgeon should attempt to maximize the extent of removal without compromising the patient's quality of life. 
Transcranial magnetic stimulation (TMS) is a relatively recent and promising method for both investigation and therapeutic treatment of psychiatric and neurologic disorders. ${ }^{5-8} \mathrm{In}$ this method, small volumes of brain tissue close to the cortical surface can be activated by a single pulsed magnetic field, thereby providing an emerging method for noninvasive brain mapping. ${ }^{9-13}$ However, despite technical advances in TMS, practical application of this method remains limited because of inaccuracies in localization of the exact area controlling the behavioral response. A solution to the problem of coil positioning can be provided by integration of TMS and the navigation devices currently used in neurosurgery. Navigated transcranial magnetic stimulation (nTMS) is the only painless noninvasive method that allows for functional testing analogous to the procedure of direct cortical electrical stimulation (DES). The purpose of this study is to report the application of preoperative nTMS mapping and its spatial correlation to intraoperative navigated cortical mapping, based on direct current stimulation, during surgical resection of low-grade gliomas (LGGs) located in or near the motor cortex.

\section{Materials and methods Experimental procedure}

This prospective study included six patients with LGGs in or near the motor cortex (Table 1). This study was approved by our institutional ethics committee (CAPPEsq - Ethics Committee for Analysis of Research Projects of the University of São Paulo Medical School, São Paulo, Brazil) and in accordance with the Declaration of Helsinki. All the patients underwent high-field magnetic resonance imaging (MRI). Initially, a sensor-based navigation system was used for guidance of coil placement, which allowed projection of the focus and visualization of the angle of impact for the magnetic impulse onto the cortical surface. nTMS is a new brain-mapping modality that combines the anatomic accuracy provided by MRI and the functional motor specificity provided by TMS. Subsequently, a system was introduced that also allowed for display of the exact strength and extent of the induced electrical field, depending on the depth of the area under the coil and the angle of tilt and rotation of the coil. Intraoperatively, direct cortical electrical stimulation was performed in all cases. In addition to the structurefunction correlation of TMS, this technique further allowed for integration of different brain mapping methodologies by providing a common coordinate system for DES maps. Motor activity was measured from the abductor pollicis brevis muscle. During TMS stimulation, electromyography was recorded using surface electrodes.

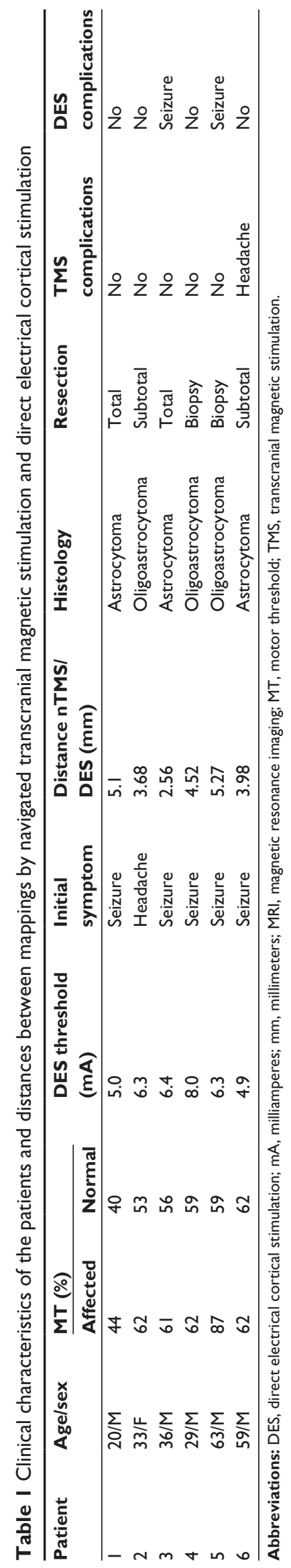


In all patients, MRI showed tumor lesions adjacent to the motor cortex (tumor volume range: $6.8-14.2 \mathrm{~cm}^{3}$ ). T1-weighted MRI scans (magnetization-prepared radiofrequency pulses and rapid gradient-echo [MPRAGE]; voxel size, $1 \mathrm{~mm}^{3}$ ) were acquired using a $1.5 \mathrm{~T}$ Magnetom Vision MR scanner (Siemens, Berlin, Germany). In each case, evaluation of the tumor location led to the decision to perform cortical mapping with nTMS. The nTMS was performed 1 day before surgery.

During nTMS, the patient sat in a comfortable bed with a headrest. Focal single-pulse TMS was delivered to the left motor cortex through a polyfoam-coated figure-of-eight coil (diameter $70 \mathrm{~mm}$; nine turns of wire; peak magnetic field strength, 2.2 T; peak electric field strength, $660 \mathrm{~V} / \mathrm{m}$ ). The magnetic coil was positioned using neuronavigation based on optical tracking. The primary motor cortical area was identified using TMS applied to the cortex to induce movement in the contralateral hand. The stimulation intensity was $120 \%$ of the motor threshold. For neuronavigation, a BrainSight system (BrainSight 2; Rogue Research Inc, Montreal, Canada) was used for real-time visualization of the coil location in relation to certain predefined facial landmarks. For spatial detection of the coil with respect to the head, the navigator device uses light-emitting diodes. After coregistration, the patient's head and the MRI scan were represented in a common coordinate system, such that the position of the coil with respect to the brain could be visualized on the corresponding MRI slice shown by the navigator.

\section{Surgical procedure}

The surgery was performed with a neuronavigation system with three-dimensional MRI for skin incision and craniotomy, exposing tumor limits and motor cortex. The classical microsurgical technique was applied and was associated with DES in order to determine the motor cortex limits. DES at the time of surgery was performed with a hand-held constant-current bipolar stimulator (Micromar, São Paulo, Brazil) at $50 \mathrm{~Hz}$, using a biphasic square wave and a pulse width of $1.0 \mathrm{~ms}$. This stimulation tool was recognized by the navigation system, and the locations at which the desired motor response was elicited were acquired for map construction (Figure 1). Stimulation was performed broadly over the exposed cortical surface. The acquired DES locations overlapped with the locations obtained with nTMS (Figure 2).

\section{Results}

Of the six patients, four were located in precentral gyrus and had functional involvement of the motor area in the two mapping methods. In these cases, we performed two subtotal resections and in two patients we performed a biopsy only. Mean distance from tumor to central sulcus was $1.85 \mathrm{~mm}$ (range: 0-3.2 $\mathrm{mm}$ ) and mean distance from tumor to the motor cortex (defined by DES mapping) was $3.6 \mathrm{~mm}$. No patient had preoperative motor deficit. In two patients motor deficit worsened in the immediate postoperative period, but they completely recovered from the deficit at 3 months follow-up.

Both TMS and DES systems allowed cartographical analysis of peritumoral cortical motor function. Motor cortical area mapping was achieved in all patients by using TMS and DES. nTMS allowed localization of the actual stimulation site in the motor cortex, avoiding variation induced by morphologic deviation and shift due to tumor compression. The average motor threshold by using TMS was

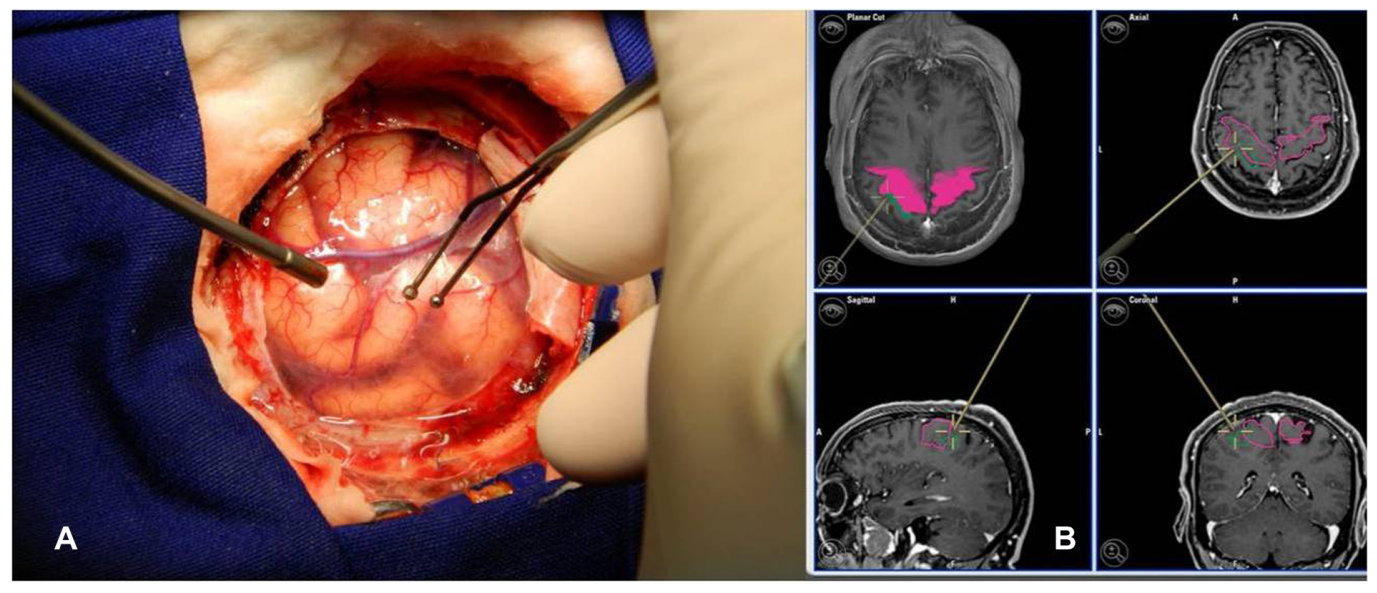

Figure I (A) Mapping with DES after craniotomy with a bipolar-stimulation-system-like neuronavigation tool. (B) Neuronavigation system image corresponding to this point in DES mapping in a three-dimensional, axial, sagittal, and coronal slice.

Abbreviation: DES, direct electrical cortical stimulation. 


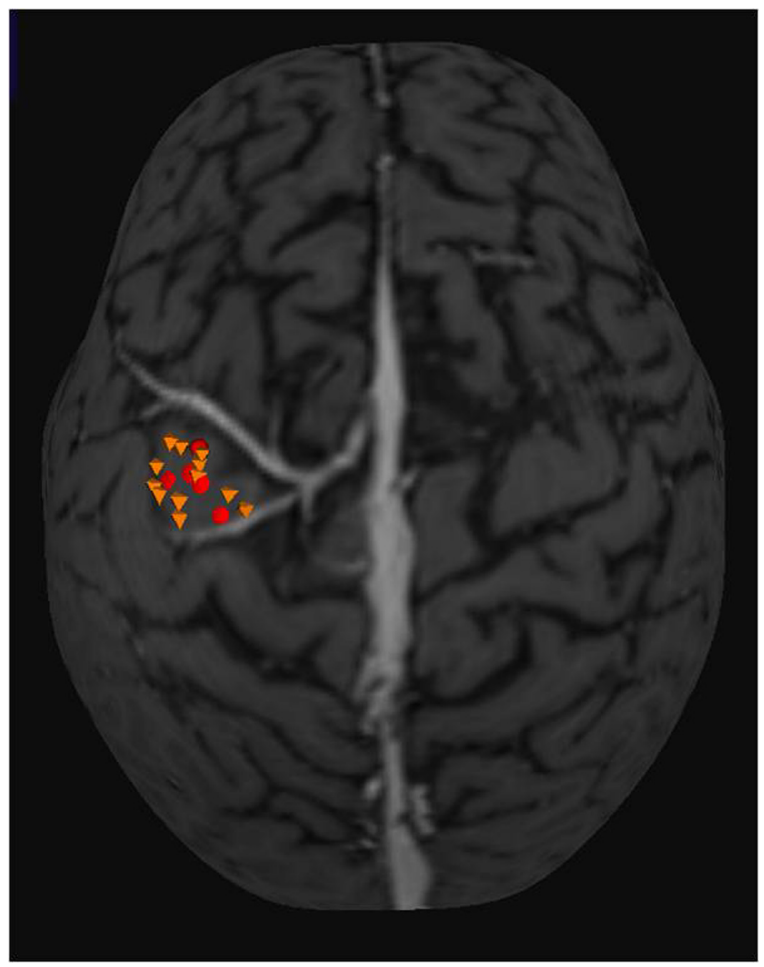

Figure 2 Three-dimensional MRI used in neuronavigation system. Yellow octahedrons are points with motor response recorded in the TMS mapping. Red circles are the points with the motor response in DES mapping.

Note: The mean distance between the two mappings found was $4.16 \mathrm{~mm}$.

Abbreviations: DES, direct electrical cortical stimulation; MRI, magnetic resonance imaging; TMS, transcranial magnetic stimulation.

$63 \% \pm 13.74 \%$ (range: $44 \%-87 \%$ ) of the stimulator output. Using navigation, DES (4.9-8.0 mA, parallel arrangement of electrodes) identified five to 18 points with similar responses, corresponding to motor activity elicited by TMS (Table 1). The TMS and DES hotspots were located on the same gyrus in all cases. The mean distance of geometric center of TMS mapping and DES mapping was $4.16 \pm 1.02 \mathrm{~mm}$ (range: 2.56-5.27 mm). Care was taken to identify the motor cortex both before and during tumor removal.

Two patients had a seizure during intraoperative DES, which was quickly relieved with diazepam and cold physiologic saline solution. No persistent postoperative neurologic or clinical morbidities were identified.

\section{Discussion}

Localization of the primary motor cortex is an important neurosurgical consideration when approaching lesions in motor areas. DES at the time of surgery allows precise identification of the motor cortex, but cannot provide information preoperatively for surgical planning. ${ }^{14,15}$ The accuracy of the navigational method is within millimeters and can be easily controlled by using defined anatomical landmarks. ${ }^{14}$
The accuracy depends on the resolution of the structural MRI, the functional neuroimaging (if performed), the accuracy of the referencing procedure of the head in space and of the head during MRI, and on the properties of the electric field and its effect on the cortex. ${ }^{16}$ Slices in the T1-weighted MRI scan were used in the current study.

The main advantage of nTMS is that it allows investigators to align the center of the figure-of-eight coil with the target site and to monitor all degrees of freedom of the coil, including the angle of the coil on the scalp, with great topographic precision in the motor cortex. ${ }^{17-19}$ The clinical utility of TMS mapping in brain tumor surgery has recently been evaluated..$^{20,21}$ Krings et $\mathrm{a}^{20}{ }^{20}$ reported two patients who had meningiomas and underwent mapping with nTMS and described the mapping protocol as noninvasive and free of known risks, suggesting that electrophysiologic data can readily be obtained noninvasively from both normal subjects and patients. Thus, a possible role for the nTMS mapping technique is the assessment of motor reorganization. In our study, an analogous procedure was used by employing the contralateral motor hand region as a reference location for the placement of the coil above the homotopic brain area; however, the mapping in this study was performed with respect to a primary brain tumor.

Herwig et $\mathrm{al}^{21}$ described the use of nTMS in normal subjects and found that this method had improved accuracy when compared to other TMS positioning techniques. Consequently, nTMS positioning appears to be the most appropriate method to accurately examine and preoperatively define specific cortical regions for future brain mapping studies or treatment interventions. In this study, the localization was confirmed via DES during open brain microsurgery, confirming the precision of the method.

This study represents the first investigation in a sample of patients without major anatomical deformity of the precentral gyrus. Picht et $\mathrm{al}^{22}$ and Forster et $\mathrm{al}^{23}$ have described mapping of the motor cortex in patients with growing malignant and large tumors. The localization error between TMS and DES in these studies was $7.83 \mathrm{~mm}$ and $10.5 \mathrm{~mm}$, respectively. However, in these situations, the mass effect must be taken into account. In the current study, an average localization error of $4.16 \mathrm{~mm}$ was found. It is likely that the improved precision found in the current study is the result of a more homogeneous sample (ie, only patients with LGGs were included; largest tumor diameter, $4 \mathrm{~cm}$ ).

TMS seems to be a useful presurgical planning tool for noninvasive motor cortex mapping in LGG surgery. This technique is a promising option for use during surgery of brain tumors. 


\section{Disclosure}

The authors report no conflicts of interest in this work.

\section{References}

1. Kim J, Horwitz B. Investigating the neural basis for fMRI-based functional connectivity in a blocked design: application to interregional correlations and psycho-physiological interactions. Magn Reson Imaging. 2008;26(5):583-593

2. Dolbakyan EE, Merzhanova GKh. Organization of neural networks in the neocortex. Neurosci Behav Physiol. 2003;33(6):555-565.

3. Säisänen L, Pirinen E, Teitti S, et al. Factors influencing cortical silent period: optimized stimulus location, intensity and muscle contraction. J Neurosci Methods. 2008;169(1):231-238.

4. Herwig U, Schönfeldt-Lecuona C, Wunderlich AP, et al. The navigation of transcranial magnetic stimulation. Psychiatry Res. 2001;108(2):123-131.

5. Saitoh Y, Hirayama A, Kishima H, et al. Reduction of intractable deafferentation pain due to spinal cord or peripheral lesion by highfrequency repetitive transcranial magnetic stimulation of the primary motor cortex. J Neurosurg. 2007;107(3):555-559.

6. Fregni F, Ono CR, Santos CM, et al. Effects of antidepressant treatment with rTMS and fluoxetine on brain perfusion in PD. Neurology. 2006;66(11):1629-1637.

7. Fregni F, Boggio PS, Nitsche MA, Marcolin MA, Rigonatti SP, PascualLeone A. Treatment of major depression with transcranial direct current stimulation. Bipolar Disord. 2006;8(2):203-204.

8. Minichino A, Bersani FS, Capra E, et al. ECT, rTMS, and deepTMS in pharmacoresistant drug-free patients with unipolar depression: a comparative review. Neuropsychiatr Dis Treat. 2012;8:55-64.

9. O’Connell NE, Maskill DW, Cossar J, Nowicky AV. Mapping the cortical representation of the lumbar paravertebral muscles. Clin Neurophysiol. 2007;118(11):2451-2455.

10. Tyc F, Boyadjian A, Devanne H. Motor cortex plasticity induced by extensive training revealed by transcranial magnetic stimulation in human. Eur J Neurosci. 2005;21(1):259-266.

11. Barker AT, Jalinous R, Freeston IL. Non-invasive magnetic stimulation of human motor cortex. Lancet. 1985;1(8437):1106-1107.
12. Barker AT. The history and basic principles of magnetic nerve stimulation. Electroencephalogr Clin Neurophysiol Suppl. 1999;51:3-21.

13. Rossini PM, Rossi S. Transcranial magnetic stimulation: diagnostic, therapeutic, and research potential. Neurology. 2007;68(7):484-488.

14. Carrabba G, Fava E, Giussani C, et al. Cortical and subcortical motor mapping in rolandic and perirolandic glioma surgery: impact on postoperative morbidity and extent of resection. J Neurosurg Sci. 2007;51(2):45-51.

15. Sala F, Lanteri P. Brain surgery in motor areas: the invaluable assistance of intraoperative neurophysiological monitoring. J Neurosurg Sci. 2003;47(2):79-88.

16. Neggers SF, Langerak TR, Schutter DJ, et al. A stereotactic method for image-guided transcranial magnetic stimulation validated with fMRI and motor-evoked potentials. Neuroimage. 2004;21(4):1805-1817.

17. Sparing R, Buelte D, Meister IG, Paus T, Fink GR. Transcranial magnetic stimulation and the challenge of coil placement: a comparison of conventional and stereotaxic neuronavigational strategies. Hum Brain Mapp. 2008;29(1):82-96.

18. Krings T, Foltys H, Reinges MH, et al. Navigated transcranial magnetic stimulation for presurgical planning - correlation with functional MRI Minim Invasive Neurosurg. 2001;44(4):234-239.

19. Schönfeldt-Lecuona C, Thielscher A, Freudenmann RW, Kron M, Spitzer M, Herwig U. Accuracy of stereotaxic positioning of transcranial magnetic stimulation. Brain Topogr. 2005;17(4):253-259.

20. Krings T, Chiappa KH, Foltys H, Reinges MH, Cosgrove GR, Thron A. Introducing navigated transcranial magnetic stimulation as a refined brain mapping methodology. Neurosurg Rev. 2001;24(4):171-179.

21. Herwig U, Satrapi P, Schönfeldt-Lecuona C. Using the international 10-20 EEG system for positioning of transcranial magnetic stimulation. Brain Topogr. 2003;16(2):95-99.

22. Picht T, Schmidt S, Brandt S, Frey D, et al. Preoperative functional mapping for rolandic brain tumor surgery: comparison of navigated transcranial magnetic stimulation to direct cortical stimulation. Neurosurgery. 2011;69(3):581-588

23. Forster MT, Hattingen E, Senft C, Gasser T, Seifert V, Szelényi A. Navigated transcranial magnetic stimulation and functional magnetic resonance imaging: advanced adjuncts in preoperative planning for central region tumors. Neurosurgery. 2011;68(5):1317-1324.
Neuropsychiatric Disease and Treatment

\section{Publish your work in this journal}

Neuropsychiatric Disease and Treatment is an international, peerreviewed journal of clinical therapeutics and pharmacology focusing on concise rapid reporting of clinical or pre-clinical studies on a range of neuropsychiatric and neurological disorders. This journa is indexed on PubMed Central, the 'PsycINFO' database and CAS.

\section{Dovepress}

The manuscript management system is completely online and includes a very quick and fair peer-review system, which is all easy to use. Visit http://www.dovepress.com/testimonials.php to read real quotes from published authors. 